Samuel S.H.

Nagoya Math. J.

Vol. 33 (1968), 165-172

\title{
ON THE SUM OF \\ HOMOLOGICAL DIMENSION AND CODIMENSION OF MODULES OVER A SEMI-LOCAL RING
}

\author{
SAMUEL S.H. YOUNG
}

\section{Introduction.}

Let $M$ be a finitely generated module over a regular local ring $R$. It is well known that the sum of homological dimension and codimension of $M$ is equal to the global dimension of $R$. For modules over an arbitrary ring, this is in general not true. The purpose of this paper is to investigate the properties of such sums in the semi-local case.

Throughout this paper, we shall use $S$ to denote a semi-local ring, that is, a commutative noetherian ring with unity having only a finite number of maximal ideals. We shall also assume that $S$ is of finite global dimension and that all $S$-modules are non-null, finitely generated and unitary. Known results are as a rule quoted without proof.

In Section 1, we collect some results concerning $M$-sequences and codimension. In Section 2, we generalize a proposition relative to local rings to the semi-local case. In Section 3, we define the pro-global and pro-total dimensions of $S$-modules and the total dimension of the ring $S$ itself. Section 4 is concerned with the relations between the various dimensions. In Section 5, we show that the various dimensions remain unchanged on completion. The results are then applied in Section 6 to study the characterization of semi-local rings of total dimension 2.

Thanks are due to Professor Y.C. Wong for his encouragement and support and to Dr. K.T. Leung for first stimulating the author's interest in the theory of local rings and homology.

\section{M-Sequences and Codimension.}

Definition 1. 1. Let $M$ be a finitely generated module over a noetherian ring $R$. A sequence of elements $\left\{u_{1}, \cdots, u_{t}\right\}$ in an ideal $\mathfrak{i t}$ of $R$ is called an $M$-sequence in $\mathfrak{n}$ if $u_{i+1}$ is not a zero divisor of $M /\left(u_{1}, \cdots, u_{i}\right) M$

Received January 22, 1968. 
for $1 \leqslant i<t$ and $M /\left(u_{1}, \cdots, u_{t}\right) M \neq 0$. The sequence is said to be maximal if there does not exist any element $u_{t+1} \in \mathfrak{i t}$ such that $\left\{u_{1}, \cdots, u_{t}, u_{t+1}\right\}$ is an $M$-sequence in $\mathfrak{i t}$.

Note. In the sequel, we shall write $M_{(i)}$ and $R_{(i)}$ to stand for $M /\left(u_{1}\right.$, $\left.\cdots, u_{i}\right) M$ and $R /\left(u_{1}, \cdots, u_{i}\right)$ respectively. The symbol $\left(u_{1}, \cdots, u_{i}\right)$ denotes the ideal generated by the $u$ 's in $R$.

Let $\mathfrak{m}$ be a maximal ideal of $R$. It is known that every $M$-sequence in $\mathfrak{m}$ can be extended to a maximal $M$-sequence in $\mathfrak{m}$ and that all such maximal $M$-sequences in $\mathfrak{m}$ have the same length [12]. This leads to the following definition of codimension as given by Auslander and Buchsbaum [3].

Definitron 1. 2. The common maximal length of $M$-sequences in $\mathfrak{m}$ is called the codimension of $M$ in $\mathfrak{m}$ and is denoted by $\mathfrak{m}$-codim $\cos _{R} M$. The supremum of $\mathfrak{m}$ - $\operatorname{codim}_{R} M$, where $\mathfrak{m}$ ranges over all maximal ideals of $R$ is called the codimension of $M$ and is denoted by $\operatorname{codim}_{R} M$.

Let $S$ be a semi-local ring with Jacobson radical $\mathfrak{r}$. For any $S$-module $N, \mathfrak{r}$ - $\operatorname{codim}_{S} N$ is equal to the smallest integer $p$ such that $\operatorname{Ext}_{S}^{p}(S / \mathfrak{r}, N) \neq 0$ (see [5], Prop. 2. 9, Cor. 2. 10, for instance). Thus, $r$ - $\operatorname{codim}_{S} N$ is just the grade of $N$ as first introduced by Rees [11]. Since the properties of $\mathfrak{r}$ $\operatorname{codim}_{S} N$ can be easily derived by using the functor $\operatorname{Ext}_{S}^{p}(S / \mathfrak{r},-)$, it is more convenient, in the semi-local case, to replace Defn. 1.2 by the following definition as given by Serre [13].

Definition 1.3. The common maximal length of $N$-sequences in $\mathfrak{r}$ is called the homological codimension of $N$ and is denoted by $\operatorname{cohd}_{S} N$.

We list without proof a few propositions which will be used later.

Proposition 1. 4. Let $N$ be an S-module. Then

(i) $\operatorname{codim}_{S} N=\sup _{\mathfrak{m}} \operatorname{codim}_{S_{\mathfrak{m}}} N_{\mathrm{m}}, \quad$ ([3], Thm. 1. 4)

(ii) $\operatorname{cohd} d_{S} N=\inf _{\mathfrak{m}} \operatorname{cohd} d_{S_{\mathfrak{m}}} N_{\mathfrak{m}}, \quad$ ([13], Ch. IV, Prop. 9)

where $\mathfrak{m}$ ranges over all maximal ideals of $S$.

Note. $\quad N_{\mathfrak{m}}$ and $S_{\mathfrak{m}}$ denote respectively the module of fractions and the ring of fractions of $N$ and $S$ with respect to $\mathfrak{m}$. 
Proposition 1.5. Let $\left\{u_{1}, \cdots, u_{t}\right\}$ be a maximal $N$-sequence (in (ii) below, the sequence is maximal in $\mathfrak{r})$. Then for $1 \leqslant i \leqslant t$, we have

(i) $\operatorname{codim}_{S} N=\operatorname{codim}_{S_{(i)}} N_{(i)}+i, \quad$ ([3], Prop. 1. 11)

(ii) $\operatorname{cohd}_{S} N=\operatorname{cohd}_{S} N_{(i)}+i$ ([13], Ch. IV, Cor. to Prop. 6)

\section{Generalization of a Proposition Relative to Local Rings to Semi-} Local Case.

Let $N$ be an $S$-module. There exists a maximal ideal $\mathfrak{h}$ of $S$ such that

$$
\operatorname{hd}_{S_{\mathfrak{h}}} N_{\mathfrak{h}}=\operatorname{hd}_{S} N .
$$

If $\operatorname{hd}_{S} N=p$, then since $S_{\mathfrak{h}}$ is a local ring, we have

$$
\operatorname{Tor}_{p} s_{\mathfrak{h}}\left(N_{\mathfrak{h}}, K_{\mathfrak{h}}\right) \neq 0 \text {, }
$$

where $K$ denotes the residue field $S / \mathfrak{h}$ ([10], Ch. 9, Thm. 11). Using this fact, a useful proposition relative to local rings ([2], Prop. 1. 4) can be extended to the semi-local case as follows:

Proposition 2. 1. Let $\left\{u_{1}, \cdots, u_{t}\right\}$ be a maximal $N$-sequence in the Jacobson radical $\mathfrak{r}$ of $S$. Then for $1 \leqslant i \leqslant t$, we have

$$
h d_{S} N_{(i)}=h d_{S} N+i \text {. }
$$

Proof. Since $N=\mathrm{r} N$ implies $N=0$, we see that $N_{(i)} \neq 0$ for each $i$. Furthermore, $N_{(i)}$ is finitely generated and $N_{(i)}=u_{i} N_{(i-1)}$. Thus, we need only prove the proposition for the case $i=1$, of which the general case is a simple consequence.

Consider the exact sequence

$$
0 \longrightarrow N \stackrel{f}{\rightarrow} N \rightarrow N / u_{1} N \longrightarrow 0
$$

where $f$ is multiplication by $u_{1} \in \mathfrak{h}$, which is not a zero divisor of $N$. This gives rise to an exact sequence

$$
\begin{aligned}
\cdots \cdots \rightarrow & \operatorname{Tor}_{q+1}^{S}(N, K) \stackrel{f_{q+1}}{\rightarrow} \operatorname{Tor}_{q+1}^{S}(N, K) \rightarrow \rightarrow \operatorname{Tor}_{q+1}^{S}\left(N / u_{1} N, K\right) \\
& \cdots
\end{aligned}
$$

where $K$ has the meaning as already given. Since $u_{1} K=0, f_{q}$ is a null map for every $q \geqslant 0$. Hence, the sequence 


$$
0 \longrightarrow \rightarrow \operatorname{Tor}_{q+1}^{S}(N, K) \rightarrow \operatorname{Tor}_{q+1}^{S}\left(N / u_{1} N, K\right) \rightarrow \longrightarrow \operatorname{Tor}_{q}^{S}(N, K) \longrightarrow 0
$$

is also exact. Assume that $\mathrm{hd}_{S} N=p$. Then the middle term of $(*)$ is not zero for $q=p$. For if otherwise, we would lead to $\operatorname{Tor}_{p}^{S}(N, K)=0$, and consequently

$$
\operatorname{Tor}_{p}^{S_{\mathfrak{h}}}\left(N_{\mathfrak{h}}, K_{\mathfrak{h}}\right) \cong\left(\operatorname{Tor}_{p}^{S}(N, K)\right)_{\mathfrak{h}}=0_{\mathfrak{h}}=0,
$$

which is not true. (For the proof of the above isomorphism, see [10], Ch. 8, Thm. 7). Hence, $\operatorname{hd}_{S}\left(N / u_{1} N\right) \geqslant p+1$.

Replacing $K$ by an arbitrary $S$-module $M$ in (*), we see that $\operatorname{Tor}_{p+2}^{s}\left(N / u_{1} N, M\right)=0$ for $q=p+1$. It follows that $\operatorname{hd}_{S}\left(N / u_{1} N\right) \leqslant p+1$, and accordingly $\operatorname{hd}_{S}\left(N / u_{1} N\right)=\operatorname{hd}_{S} N+1$ as desired.

\section{Notions of Pro-Global, Pro-Total and Total Dimensions.}

By combining Prop. 2. 1 with Prop. 1. 5, we obtain the following

Proposition 3. 1. Let $\left\{u_{1}, \cdots, u_{t}\right\}$ be a maximal $N$-sequence in $\mathfrak{r}$. Then for $0 \leqslant i<j \leqslant t$, we have

(i) $\quad h d_{S} N_{(i)}+\operatorname{codim}_{S_{(i)}} N_{(i)}=h d_{S} N_{(j)}+\operatorname{codim}_{S_{(j)}} N_{(j)}$,

(ii) $\quad h d_{S} N_{(i)}+\operatorname{cohd}_{S} N_{(i)}=h d_{S} N_{(j)}+\operatorname{cohd}_{S} N_{(j)}$

where by $N_{(0)}$ and $S_{(0)}$ we mean $N$ and $S$ respectively.

Definition 3. 2. The common sum of Prop. 3. 1 (i) is called the prototal dimension of $N$ and is denoted by $\operatorname{ptd}_{S} N$. That of Prop. 3. 1 (ii) is called the pro-global dimension of $N$ and is denoted by $\operatorname{pgd}_{S} N$.

Definition 3. 3. The supremum of $\operatorname{ptd}_{S} N$, where $N$ ranges over all $S$-modules, is called the total dimension of $S$ and is denoted by $\mathrm{Td} S$.

\section{Relations Between Various Dimensions.}

Proposition 4. 1. The following inequalities hold for any S-module $N$ :

$$
p t d_{S} N \geqslant G d S \geqslant p g d_{S} N
$$

where $G d S$ denotes the global dimension of $S$.

Proof. The inequality on the right follows from the fact that if $\left\{u_{1}\right.$, $\left.\cdots, u_{t}\right\}$ is a maximal $N$-sequence in $r$, then $\operatorname{cohd}_{S} N_{(t)}=0$, and hence $\operatorname{pgd}_{S} N=\operatorname{hd}_{S} N_{(t)} \leqslant \mathrm{Gd} S$. 
To establish the inequality on the left, we have

$$
\begin{aligned}
\operatorname{ptd}_{S} N & =\sup _{\mathfrak{m}} \operatorname{hdd}_{S_{\mathfrak{m}}} N_{\mathfrak{m}}+\sup _{\mathfrak{m}} \operatorname{codim}_{S_{\mathfrak{m}}} N_{\mathfrak{m}} \\
& \geqslant \sup _{\mathfrak{m}}\left(\operatorname{hd}_{S_{\mathfrak{m}}} N_{\mathfrak{m}}+\operatorname{codim}_{S_{\mathfrak{m}}} N_{\mathfrak{m}}\right) \\
& =\sup _{\mathfrak{m}} \operatorname{Gd} S_{\mathfrak{m}}=\mathrm{Gd} S .
\end{aligned}
$$

Lemma 4. 2. For every semi-local ring $S$ of finite global dimension, we have Gd $S=\operatorname{codim}_{S} S$.

Proof. Since for every maximal ideal $\mathfrak{m}$ of $S, S_{\mathfrak{m}}$ is a regular local ring, we have $\operatorname{Gd} S_{\mathfrak{m}}=\operatorname{codim}_{S_{\mathfrak{m}}} S_{\mathfrak{m}}$. Hence,

$$
\operatorname{Gd} S=\sup _{\mathfrak{m}} \operatorname{Gd} S_{\mathfrak{m}}=\sup _{\mathfrak{m}} \operatorname{codim}_{S_{\mathfrak{m}}} S_{\mathfrak{m}}=\operatorname{codim}_{S} S .
$$

Proposition 4. 3. A semi-local ring $S$ is a local ring iff

$$
\text { Gd } S=\operatorname{pgd}_{S} S \text {. }
$$

Proof. Necessity is obvious. To prove sufficiency, assume that $S$ has more than one maximal ideal and that $\operatorname{Gd} S=\operatorname{pgd}_{S} S=t$. By Lemma 4. 2 and observing that $\operatorname{pgd}_{S} S=\operatorname{cohd}_{S} S$, we have $\operatorname{codim}_{S} S=\operatorname{cohd}_{S} S$, which means that every maximal $S$-sequence $\left\{u_{1}, \cdots, u_{t}\right\}$ in $\mathfrak{r}$ is also maximal in any maximal ideal $\mathfrak{m}$ of $S$. Clearly, $S_{(i)} \neq 0$ for $1 \leqslant i \leqslant t$. Thus, for every $\mathfrak{m}$, all elements in $\mathfrak{m} \mid \mathfrak{r}$ are zero divisors of $S_{(t)}$. Let $\mathfrak{m}_{1}$ and $\mathfrak{m}_{2}$ be two distinct maximal ideals of $S$. Since $\mathfrak{m}_{1}$ and $\mathfrak{m}_{2}$ are comaximal, we can choose $v_{i} \in \mathfrak{m}_{i} \backslash \mathfrak{r}, \quad i=1,2$, so that the ideal generated by $v_{1}$ and $v_{2}$ in $S$ is the whole ring. But this cannot be true, for $S$, being a ring with unity which contains $\left(u_{1}, \cdots, u_{t}\right)$ properly, cannot consist entirely of zero divisors of $S_{(t)}$.

Proposition 4. 4. Let $S$ be a semi-local ring having maximal ideals $\mathfrak{m}_{1}$ and $\mathfrak{m}_{2}$ satisfying the condition

$$
\mathfrak{m}_{2}-\operatorname{codim}_{S} S \geqslant \mathfrak{m}_{1}-\operatorname{codim}_{S} S>\operatorname{cohd} d_{S} S .
$$

Then $T d S>G d S$.

Proof. Let $\left\{u_{1}, \cdots, u_{t}\right\}$ be a maximal $S$-sequence in $\mathfrak{r}$ and $\left\{u_{1}, \cdots\right.$, 
$\left.u_{t}, u\right\}$ an extension of the sequence in $m_{1}$. Then $\operatorname{codim}_{S}(S /(u))=\operatorname{codim}_{S} S$. On the other hand, $\operatorname{hd}_{S}(S /(u))=1+\operatorname{hd}_{S}(u) \geqslant 1$, since $(u) \neq(0)$. Hence, $\operatorname{Td} S \geqslant \operatorname{ptd}_{S}(S /(u)) \geqslant \operatorname{codim}_{S} S+1>\operatorname{Gd} S$.

Remark. From the proof of Prop. 4. 3, it is clear that there exists at most one maximal ideal $\mathfrak{m}$ with $\mathfrak{m}$-codim $\cos _{S} S=\operatorname{cohd}_{S} S$. Hence, the condition of Prop. 4. 4 is always satisfied except in the case when $S$ has exactly two maximal ideals, one of which, say $\mathfrak{m}$, has the property that $\mathfrak{m}$ - $\operatorname{codim}_{S} S=$ $\operatorname{cohd}_{S} S$.

Corollary 4. 5. For any semi-local ring $S$ with more than two maximal ideals, we have

$$
T d S>G d S>\operatorname{coh} d_{S} S .
$$

\section{Effect of Completion on Various Dimensions.}

In a semi-local ring $S$ (resp. an $S$-module $N$ ), a topology can be introduced by taking the powers of the Jacobson radical $\mathfrak{r}^{n}\left(\operatorname{resp} . \mathfrak{r}^{n} N\right)$, $n=0,1,2, \cdots$, to be the neighbourhoods of 0 . This is the natural topology of $S$ (resp. $N$ ) with respect to which we can construct the completion $\hat{S}$ (resp. $\hat{N}$ ) of $S$ (resp. $N$ ). It is well known that the homological dimension of any $S$-module $N$ remains unchanged on completion, and hence also $\operatorname{Gd} S=\operatorname{Gd} \hat{S}$ ([13], Ch. IV, Cor. 1 to Prop. 18). Furthermore, we have $\operatorname{codim}_{S} N=\operatorname{codim}_{\hat{S}} \hat{N}$ and $\operatorname{cohd}_{S} N=\operatorname{cohd}_{\hat{S}} \hat{N}$ ([13], Ch. IV, Prop. 8). We can thus easily deduce the following

Proposition 5. 1. For any $S$-module $N$, we have

$$
p t d_{S} N=p t d_{\hat{S}} \hat{N} \quad \text { and } \quad p g d_{S} N=p g d_{\hat{S}} \hat{N} .
$$

By taking the supremum over all $S$-modules $N$ in the first equality above, we obtain

Proposition 5. 2. For any S-module $N$, we have

$$
T d S=T d \hat{S} \text {. }
$$

\section{Semi-Local Rings of Total Dimension 2.}

Proposition 6. 1. Let $S$ be a semi-local ring with more than two maximal 
ideals and $T d S=2$. Then its completion $\hat{S}$ is isomorphic to the direct sum of complete principal ideal domains.

Proof. It is known that the completion $\hat{S}$ of $S$ is isomorphic to the direct sum of $\hat{S}_{\mathfrak{m}}$, where $\mathfrak{m}$ ranges over all maximal ideals of $S$ ([8], Ch. II, Thm. 17. 7). We proceed to investigate the properties of $\hat{S}_{\mathfrak{m}}$.

For every $\mathfrak{m}, S_{\mathfrak{m}}$ is a regular local ring since $\operatorname{Gd} S$ is assumed to be finite in this paper. The Krull dimension of $S_{\mathfrak{m}}$ (to be denoted by Dim $S_{\mathfrak{m}}$ ) is thus the same as that of its completion and we have

$$
\operatorname{Dim} \hat{S}_{\mathrm{m}}=\operatorname{Dim} S_{\mathrm{m}}=\operatorname{Gd} S_{\mathrm{m}} \leqslant \operatorname{Gd} S=1,
$$

since $2=\mathrm{Td} S>\operatorname{Gd} S>0$ (Cor. 4. 5). On the other hand, $\operatorname{Gd} S_{\mathfrak{m}}=\operatorname{hd}_{S}(S / \mathfrak{m}$ ) $=\mathrm{hd}_{S} \mathfrak{m}+1 \geqslant 1$ since $\mathfrak{m} \neq(0)$. Hence, $\hat{S}_{\mathfrak{m}}$ is a regular local ring of Krull dimension 1. Our proposition then follows from the fact that every such ring is a local domain, of which all non-zero proper ideals are principal ideals ([9], Ch. IV, Prop. 7).

Proposition 6. 2. Let $S$ be a semi-local ring with more than two maximal ideals and $T d S=2$. Then $S$ is a principal ideal ring. There is exactly one maximal ideal of $S$ which is generated by a zero divisor of $S$.

Proof. By Prop. 6. 1, $\hat{S}$ is the direct sum of principal ideal domains and is thus a principal ideal ring ([14], Vol. I, Ch. IV, Thm. 33).

For any non-zero proper ideal $\mathfrak{n}$ of $S$, we have $\mathfrak{n}=\hat{\mathfrak{t}} \cap S$ and so $\mathfrak{n}$ is also a principal ideal. Denote the maximal ideals of $S$ by $\left(v_{i}\right)$, where $i$ ranges over a finite number of indices, and let $\mathfrak{r}=(u)$. Then $u$ is a zero divisor of $S$ since $\operatorname{Td} S=2$ implies $\operatorname{cohd}_{S} S=0$. Since $\Pi_{i} v_{i} \in(u)$, at least one of the $v_{i}$ is a zero divisor of $S$. Using the arguments as given in the last part of the proof of Prop. 4. 3, at most one of the $v_{i}$ is a zero divisor of $S$. This completes the proof of our proposition.

Proposition 6. 3. Let $S$ be a semi-local ring with more than two maximal ideals. If $S$ is at the same time a principal ideal ring, then $T d S=2$.

Proof. It is easily seen that $\operatorname{Dim} S=1$ and hence $\operatorname{codim}_{S} S=\operatorname{Gd} S=1$ ([3], Cor. 1. 7). By Cor. 4. 5, we have $\mathrm{Td} S \geqslant 2$. To establish the reverse inequality, it suffices to show that $\operatorname{ptd}_{S} N \leqslant 2$ for an arbitrary $S$-module $N$. 
Since for every maximal ideal $\mathfrak{m}$ of $S, S_{\mathfrak{m}}$ is a regular local ring, we have $\operatorname{codim}_{S_{\mathfrak{m}}} N_{\mathfrak{m}}=G d S_{\mathfrak{m}} \leqslant 1$. Thus, $\operatorname{codim}_{S} N \leqslant 1$ by Prop. 1.4 (i), and so

$$
\operatorname{ptd}_{S} N=\operatorname{hd}_{S} N+\operatorname{codim}_{S} N \leqslant 1+1=2
$$

as desired.

\section{REFERENCES}

[1] M. Auslander, Remarks on a theorem of Bourbaki, Nagoya Math. J., Vol. 27-1 (1966), pp. 361-369.

[2] M. Auslander \& D.A. Buchsbaum, Homological dimension in local rings, TAMS, 85 (1957), pp. 390-405.

[ 3 ] M. Auslander \& D.A. Buchsbaum, Homological dimension in noetherian rings, II, TAMS, 88 (1958), pp. 194-206.

[4] M. Auslander \& D.A. Buchsbaum, Codimension and multiplicity, Ann. of Math., 68 (1958), pp. 625-657.

[ 5 ] H. Bass, On the ubiguity of Gorenstein rings, Math. Zeitsch., 82 (1963), pp. 8-28.

[6] H. Cartan \& S. Eilenberg, Homological algebra, Princeton, 1945.

[ 7 ] J.P. Jans, Rings and homology, Holt, Reinhart \& Winston, 1964.

[8] M. Nagata, Local rings. Interscience, 1962.

[9] D.G. Northcott, Ideal theory. Cambridge, 1953.

[10] D.G. Northcott, An introduction to homological algebra, Cambridge, 1960.

[11] D. Rees, The grade of an ideal or module, Proc. Camb. Phil. Soc., 53 (1957), pp. 28-42.

[12] J.P. Serre, Sur la dimension homologie des anneaux et des modules noétheriens, Proc. of International Symp. on Alg. Number Theory. Tokyo, 1956. pp. 175-189.

[13] J.P. Serre, Locale algèbre-multiplités. Springer-Verlag, 1965.

[14] O. Zariski, Commutative algebra, Vol. I \& II. Van Nostrand, 1958.

University of Hongkong. 